patients were excluded due to primary and secondary failure, complications, loss to 6-month follow-up, incomplete data, etc. Compared with a group of RA patients with depression remission $(n=124)$, a group of patients with no depression remission $(n=28)$ had a younger age $(p=0.000)$, female sex $(p=0.039)$, lower serum MMP-3 levels $(p=0.021)$, lower HAQ-DI $(p=0.018)$, lower HAM-D score $(p=0.000)$, and higher Role/Social component summary score of the SF-36 $(p=0.009)$ by univariate analyses. The binominal logistic analyses findings were as follows: younger age ( $p=0.0045$, odd ratio: $0.94,95 \% \mathrm{Cl}: 0.8-0.98)$, female sex ( $\mathrm{p}=0.021$, odd ratio: $0.21,95 \% \mathrm{Cl}: 0.054-0.79$ ), and lower HAM-D scores ( $\mathrm{p}=0.0073$, odd ratio: $0.85,95 \% \mathrm{Cl}: 0.76-0.96$ ).

Conclusions: It was suggested that RA patients who are female, younger in age and have lower depression scores at baseline are more likely to achieve depression remission status with the biologic treatment.

Acknowledgements: All Showa University in Rheumatoid Arthritis (ASHURA) group

Disclosure of Interest: Y. Miwa Grant/research support from: Astellas Pharma Inc., Mitsubishi Tanabe Pharma Corporation, Pfizer Japan Inc., Chugai Pharmaceutical Co., Ltd., and Eizai Co., Ltd., Ono Pharma Co., Ltd., N. Yajima: None declared, T. Isozaki: None declared, R. Takahashi: None declared, Y. Miura: None declared, Y. Ikari: None declared, M. Hatano: None declared, T. Hayashi: None declared, T. Kasama Grant/research support from: Mitsubishi Tanabe Pharma Corporation and Pfizer Japan Inc., K. Sanada: None declared DOI: 10.1136/annrheumdis-2017-eular.1443

\section{SAT0086 THE ASSOCIATION BETWEEN ELDERLY RHEUMATOID ARTHRITIS PATIENTS USING BIOLOGICS AND ADVERSE EVENTS: RETROSPECTIVE COHORT STUDY}

Y. Ikari, Y. Miwa, N. Yajima. Division of Rheumatology, Department of Medicine, Showa University School of Medicine, Hatanodai Shinagawaku Tokyo, Japan

Background: In Japan, the definition of the elderly was suggested as being over 75 years old by the Japan Geriatric Society. The effectiveness of biologics in elderly rheumatoid arthritis (RA) patients has been demonstrated in several clinical trials and cohort studies ${ }^{1}$. On the other hand, regarding safety, there are reports that the use of biologics in elderly RA patients is a significant risk for severe infections and that they do not increase the risk of serious infection ${ }^{2,3}$. There is little evidence to support the association between age of RA patients and adverse events caused by biologics.

Objectives: We aimed to examine whether the age of RA patients was associated with adverse events caused by biologics.

Methods: RA patients using biologics were eligible. The participants were collected at Showa University Hospital, Showa University Northern Yokohama Hospital and Showa University Koto Toyosu Hospital from 2005 to 2016 in a retrospective cohort study.

RA patients of 75 years and above compared with RA patients under 75 years. The primary outcome was the rate of discontinuation due to adverse events caused by biologics. Statistical analysis was Pearson's chi-square test. Multivariable analysis was performed by multi linear analysis. Covariates were sex, glucocorticoids dose, csDMARDs, interstitial pneumonia, diabetes mellitus and chronic kidney disease.

Results: In total, 309 patients were enrolled. The mean age standard deviation was $57.1 \pm 15.6$ years, and $83.4 \%$ were women. $174(56.3 \%)$ took glucocorticoid, and the mean glucocorticoid dose was $3.13 \pm 3.9 \mathrm{mg}$. The patients over 75 years were 42 patients (13.6\%), and those under 75 years were 267 patients $(86.4 \%)$. The rate of discontinuation due to adverse events caused by biologics was $11 / 42$ $(26.2 \%)$ in the patients over 75 years and over, and $21 / 267(7.9 \%)$ in the patients under the age of 75 (Relative Risk 1.24; 95\% Confidential Interval (Cl) 1.04 to $1.50 ; P$ 0.0003). Adverse events were bacterial pneumonia, pneumocystis pneumonia, exacerbation of interstitial pneumonia, urinary tract infection, herpes zoster, cytopenia, eruption, congestive heart failure. In the multivariable analysis adjusting for confounders, the rate of discontinuation in the group aged 75 years and older was significantly higher than that in the group under the age of 75 (regression coefficient $1.35 ; 95 \% \mathrm{Cl} 0.39-2.31 ; \mathrm{p}=0.006$ )

Conclusions: Our results demonstrated that the rate of discontinuation due to adverse events by biologics was high significantly in RA patients over 75 years and above.

References:

[1] Genevay S, Finckh A, Ciurea A et al. Tolerance and effectiveness of anti-tumor cecrosis factor alpha therapies in elderly patients with rheumatoid arthritis:a population-based cohort study.Arthritis Rheum 2007;57(4):679-85.

[2] Galloway JB, Hyrich KL, Mercer LK et al. Anti-TNF therapy is associated with an increased risk of serious infections in patients with rheumatoid arthritis especially in the first 6 month of treatment:updated resurts from the British Society for Rheumatology Biologics Register with special emphasis on risks in the elderly. Rheumatology (Oxford) 2011;50(1):124-31.

[3] Widdifield $\mathrm{J}$, et al. Serious infections in a population-based cohort of 86,039 serious with rheumatoid arthritis. Arthritis Care Res (Hoboken) 2013;65(3):353-61.

Disclosure of Interest: None declared

DOI: 10.1136/annrheumdis-2017-eular.5823

\section{SAT0087 THE RELEVANCE OF POOR PROGNOSTIC FACTORS FOR ACHIEVING LOW DISEASE ACTIVITY IN PATIENTS WITH RHEUMATOID ARTHRITIS: A COLLABORATIVE ANALYSIS OF THREE GERMAN COHORTS}

Y. Meissner $^{1}$, K. Albrecht ${ }^{1}$, A. Richter ${ }^{1}$, L. Baganz ${ }^{1}$, D. Huscher ${ }^{1}$

M. Schneider ${ }^{2}$, A. Strangfeld ${ }^{1}$, A. Zink ${ }^{1,3}$. ${ }^{1}$ German Rheumatism Research Center, Berlin; ${ }^{2}$ Heinrich-Heine University, Duesseldorf; ${ }^{3}$ Charité University Medicine, Berlin, Germany

Background: Poor prognostic factors (PPF) are used as decision-criteria for treatment strategies in patients with rheumatoid arthritis (RA). However, their definition is based on the outcome of rapid radiologic progression.

Objectives: To investigate the impact of PPF in RA on achieving low disease activity (LDA) at follow-up.

Methods: We performed a collaborative analysis of three large German RA cohorts. Patients under routine care were either DMARD-naïve ( $n=991$, early arthritis cohort CAPEA), on 1st conventional synthetic (cs)DMARD ( $n=2,547$, National Database of the German Arthritis Centres (NDB)), or switching to a 2nd $(n=1,959)$ or a 3rd DMARD ( $n=1,854$, both biologics register RABBIT). Disease activity based on DAS28, autoantibody positivity (RF+/ACPA+), erosions, disability $(\mathrm{HAQ} \geq 1.2)$ and intake of glucocorticoids $(>5 \mathrm{mg} / \mathrm{d})$ were evaluated as PPF at baseline. The outcome was DAS28 at $0,3,6$ and 12 months. With multinomial logistic regression analyses, predictors of either LDA (DAS28<3.2) at 12 months or treatment escalation (adding or switching to cs/biologic (b)DMARD) were investigated.

Results: Patients had a mean age of 57 to 60 years; $63 \%$ of DMARD-naïve patients and $71-72 \%$ of all others were female. Disease duration was 13 weeks in early RA (CAPEA) and 5-8 years in other cohorts. Patients with more treatment failures had more often PPF (not shown).

The figure shows the course of disease activity stratified by baseline DAS28, erosions and RF/ACPA. Values of DAS28 at baseline did not differ depending on presence/absence of erosions and autoantibodies. In all cohorts, irrespective of maintaining or escalating the initial treatment, patients with or without erosions and autoantibodies had similar DAS28 outcomes, whereas patients with moderate or high baseline DAS28 had higher disease activity during follow-up. The proportion of patients that did not achieve LDA at 12 months was $46 \%$ in DMARD-naïve, $34 \%$ on 1 st DMARD, $47 \%$ switching to 2 nd and $49 \%$ switching to 3 rd DMARD. In the multinomial model, a 1-unit increase in DAS28 was associated with a decreased probability to achieve LDA in all cohorts (table). In contrast, autoantibodies (OR from 0.8 to 1.3) and erosions (OR from 0.8 to 1.6) had no impact on achieving LDA at month 12 or on treatment escalation. The latter applied for DAS28 (OR from 0.9 to 1.5) and HAQ (OR from 0.7 to 1.2) regarding only treatment escalation.

Table 1. Selected OR $(95 \% \mathrm{Cl})$ of multinomial logistic regression

\begin{tabular}{|c|c|c|c|c|}
\hline & DMARD-naïve & 1st DMARD & 2nd DMARD & 3rd DMARD \\
\hline \multicolumn{5}{|l|}{ Predictiors of LDA } \\
\hline Age (by 5 yrs) & $0.96(0.9-1.0)$ & $0.9(0.8-0.99)$ & $0.95(0.91-0.99)$ & $0.96(0.9-1.0)$ \\
\hline DAS28 (per 1 unit) & $0.8(0.6-0.9)$ & $0.6(0.4-0.9)$ & $0.7(0.6-0.8)$ & $0.6(0.6-0.7)$ \\
\hline $\mathrm{HAQ} \geq 1.2$ & $0.9(0.6-1.3)$ & $1.1(0.6-1.9)$ & $0.6(0.5-0.8)$ & $0.6(0.4-0.7)$ \\
\hline $\mathrm{GC}>5 \mathrm{mg} / \mathrm{d}$ & $1.6(1.2-2.3)$ & $0.6(0.3-1.3)$ & $1.0(0.8-1.3)$ & $0.99(0.8-1.3)$ \\
\hline b vs. csDMARD & - & - & $1.4(1.0-1.9)$ & $1.4(1.0-1.8)$ \\
\hline \multicolumn{5}{|c|}{ Predictors for treatment escalation } \\
\hline Age (by 5 years) & $0.9(0.9-0.98)$ & $0.8(0.7-0.9)$ & $0.9(0.8-0.9)$ & $0.9(0.9-0.96)$ \\
\hline $\mathrm{GC}>5 \mathrm{mg} / \mathrm{d}$ & $1.3(0.8-1.9)$ & $4.2(1.6-11.0)$ & $1.5(1.1-2.0)$ & $1.5(1.2-2.0)$ \\
\hline
\end{tabular}

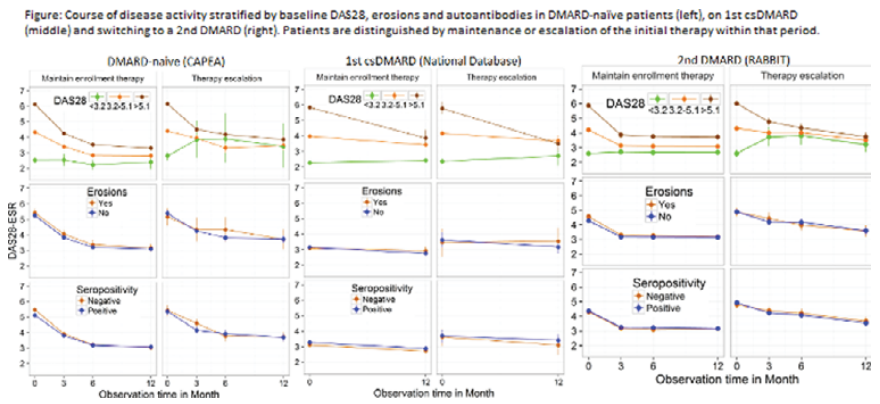

Conclusions: Although autoantibodies and erosions were associated with treatment failure, their absence did not predict LDA. Instead, baseline disease activity, functional status and glucocorticoid use may be more useful to guide treatment strategies when LDA is the target.

Disclosure of Interest: Y. Meissner Grant/research support from: CAPEA: funded by an unconditional grant from Pfizer. NDB: funded by unconditional grants from the German Collaborative Arthritis Centres and from a consortium of 11 pharmaceutical companies to the German Academy for Continuing Medical Education in Rheumatology. RABBIT: supported by a joint, unconditional grant from AbbVie, BMS, Celltrion, MSD, Pfizer, Roche, Samsung and UCB., K. Albrecht: None declared, A. Richter Consultant for: Pfizer, L. Baganz: None declared, D. Huscher: None declared, M. Schneider Grant/research support from: Abbvie, Chugai, UCB, Consultant for: Abbvie, Astra-Zeneca, BMS, Boehringer- 
Ingelheim, Chugai, Lilly, MSD, Pfizer, Roche, Sanofi-Aventis, UCB, A. Strangfeld Speakers bureau: BMS, MSD, Pfizer, Roche, Sanofi-Aventis, A. Zink Speakers bureau: AbbVie, BMS, MSD, Pfizer, Roche, UCB

DOI: 10.1136/annrheumdis-2017-eular.4697

\section{SATURDAY, 17 JUNE 2017 \\ Rheumatoid arthritis - comorbidity and clinical aspects}

\section{SAT0088 HDL CHOLESTEROL EFFLUX CAPACITY IN RHEUMATOID ARTHRITIS PATIENTS: CONTRIBUTING FACTORS AND RELATIONSHIP WITH SUBCLINICAL ATHEROSCLEROSIS}

B. Tejera Segura ${ }^{1}$, M. Macía-Díaz ${ }^{2}$, J.D.M. Machado ${ }^{2}$, A. de Vera-González ${ }^{3}$, A. González-Delgado ${ }^{3}$, J.M. Olmos ${ }^{4}$, J.L. Hernández ${ }^{4}$, F. Díaz-González ${ }^{1}$, M.A. González-Gay ${ }^{5}$, I. Ferraz-Amaro ${ }^{1} .{ }^{1}$ Division of Rheumatology;

${ }^{2}$ Pharmacology Department; ${ }^{3}$ Central Laboratory Division, Hospital Universitario de Canarias, Tenerife; ${ }^{4}$ Division of Internal Medicine; ${ }^{5}$ Rheumatology Department, Hospital Universitario Marqués de Valdecilla-IDIVAL, Santander, Spain

Background: Lipid profiles appear to be altered in rheumatoid arthritis (RA) patients due to disease activity and inflammation. Cholesterol efflux capacity (CEC) has not only been linked to cardiovascular events in the general population, but also to be impaired in RA patients.

Objectives: To analyze whether CEC is related to subclinical atherosclerosis, as determined by the presence of carotid plaque or increased levels of carotid intima-media thickness (cIMT) in RA patients. Secondarily, we aimed to describe the disease-contributing factors that are related to $C E C$ as an expression of the abnormalities in the lipid profile associated with the disease.

Methods: Cross-sectional study that encompassed 401 individuals; 178 patients with RA and 223 sex-matched controls. CEC was measured using an in vitro assay, lipoproteins serum concentrations, and standard lipid profile were assessed in patients and controls. Carotid intima-media thickness and carotid plaques were assessed in RA patients. A multivariable analysis was performed to evaluate the relation of CEC with RA-related data, lipid profile and subclinical carotid atherosclerosis.

Results: Mean CEC was not significantly different between RA patients (18.9 \pm SD $9.0 \%$ ) and controls $(16.9 \pm 10.4 \%), p=0.11$. Demographic variables were not associated with CEC except for a correlation with male gender that was only found in RA patients, but not in controls. Systolic blood pressure inversely correlated with CEC in controls (beta coefficient $-0.1[-0.2-0.0] \%, p=0.025)$. In RA patients, a similar trend was found although a statistically significant difference was not reached. Neither the traditional cardiovascular risk factors nor the cardiovascular co-morbidity-related data were associated with CEC. Similarly, lipid profile did not show any relationship with CEC in patients or controls. ESR tended to be associated with a lower CEC although it did not reach statistical significance. RA patients with low (beta coef. $-5.2[-10.0-0.3] \%, p=0.039$ ) and moderate disease activity (beta coef. $-4.6[-8.5-0.7] \%, p=0.020$ ) were associated with inferior levels of CEC when compared to patients in remission. CEC was not found to be associated with CIMT in RA patients. However, higher CEC was associated with a protective effect for the presence of carotid plaque in RA patients. This relationship was maintained even after multivariate analysis (OR 0.94 [0.89-0.98], $\mathrm{p}=0.015$ ). Conclusions: Our study, which includes the largest series of RA patients ever assessed for CEC, reveals for the first time that CEC is related to subclinical atherosclerosis in RA patients. The fact that CEC is also associated with disease activity reinforces the idea that CEC may be a mediator between disease activity and subclinical atherosclerosis.

Disclosure of Interest: None declared

DOI: 10.1136/annrheumdis-2017-eular.4100

\section{SAT0089 COMPARATIVE CARDIOVASCULAR SAFETY OF ABATACEPT AND TUMOR NECROSIS FACTOR INHIBITORS IN RHEUMATOID ARTHRITIS PATIENTS WITH AND WITHOUT TYPE 2 DIABETES: A POPULATION-BASED COHORT STUDY}

E.H. Kang ${ }^{1,2}$, Y.P. Jin ${ }^{1}$, G. Brill ${ }^{1}$, J.P. Lewey ${ }^{1,3}$, E.P. Patorno ${ }^{1}$, R. Desai ${ }^{1}$, S.C. Kim ${ }^{1} .{ }^{1}$ Division of Pharmacoepidemiology and Pharmacoeconomics, Harvard Medical School and Brigham and Women's Hospital, Boston, United States; ${ }^{2}$ Division of Rheumatology Department of Internal Medicine, Seoul National University Bundang Hospital, Seongnam, Korea, Republic Of; ${ }^{3}$ University of Pennsylvania, Philadelphia, United States

Background: Patients with rheumatoid arthritis (RA) are at high risk of developing cardiovascular disease (CVD) and may benefit from potent disease-modifying anti-inflammatory drugs such as biologics. Since diabetes mellitus (DM) is a major risk factor for CVD, RA patients with DM constitute a high CVD risk subgroup, calling for particular attention. However, there is a lack of knowledge on the comparative cardiovascular safety of different biologics in RA patients with DM. Objectives: To examine the comparative cardiovascular safety of abatacept versus TNF inhibitors in RA patients with and without DM.
Methods: RA patients enrolled in both public (Medicare) and commercial (Truven MarketScan) health plans in the U.S. who newly initiated abatacept or TNF inhibitors were eligible. The primary outcome of interest was a composite CVD endpoint of myocardial infarction (MI), stroke/transient ischemic attack (TIA), and coronary revascularization. The secondary outcomes included each component of the composite CVD endpoint and heart failure (HF). After 1:1 propensity score (PS) matching between two exposure groups, Cox proportional hazard model was used to estimate the hazard ratio (HR) and $95 \%$ confidence interval (Cl) for each outcome, comparing abatacept to TNF inhibitors. PS matching was separately done in subgroups with or without baseline DM in each database. PS-matched HRs from the two databases were pooled using an inverse variance-weighted fixed-effect model.

Results: We identified 31,899 Medicare enrollees (6,107 new users of abatacept and 25,792 new users of TNF inhibitors) and 71,956 commercial enrollees (6,942 new users of abatacept and 65,464 new users of TNF inhibitors) with RA. After PS matching, 2,119 pairs with DM and 3,984 pairs without DM were identified in Medicare and 1,371 pairs with DM and 5565 pairs without DM in MarketScan. The PS matched HR $(95 \% \mathrm{Cl})$ for the composite CVD endpoint in the whole cohort was $0.81(0.66-0.99)$ in Medicare and $0.95(0.74-1.23)$ in MarketScan with a pooled $\mathrm{HR}(95 \% \mathrm{Cl})$ of $0.86(0.73-1.01)$. In the subgroup with DM, HR $(95 \% \mathrm{Cl})$ for composite CVD endpoint was $0.72(0.53-0.99)$ in Medicare and 0.79 $(0.50-1.25)$ in MarketScan with a pooled HR $(95 \% \mathrm{Cl})$ of $0.74(0.57-0.96)$. In the subgroup without DM, HR $(95 \% \mathrm{Cl})$ was $0.88(0.67-1.14)$ in Medicare and 1.03 $(0.76-1.40)$ in MarketScan with a pooled HR $(95 \% \mathrm{Cl})$ of $0.94(0.77-1.14)$. For secondary outcomes (Figure 1), the pooled HR $(95 \% \mathrm{Cl}$ ) was $0.77(0.59-1.00)$ for $\mathrm{MI}$ and $0.74(0.57-0.97)$ for coronary revascularization in the whole cohort. There was a trend toward a decreased risk, albeit statistically insignificant, for $\mathrm{Ml}$ and coronary revascularization in each subgroup. There was no significant difference in the risk for stroke/TIA and HF

Figure 1. Cardiovascular safety of abatacept compared to TNF inhibitors

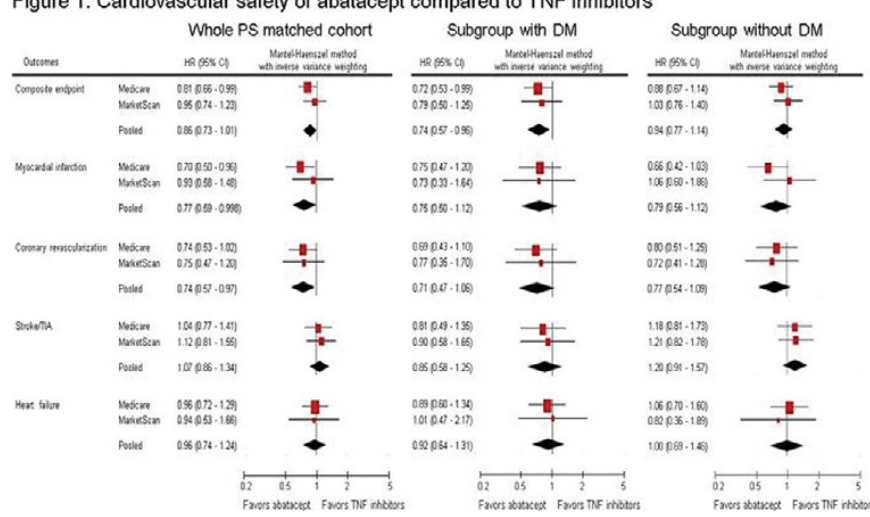

Conclusions: Among RA patients, abatacept may be associated with a reduced risk of coronary events compared to TNF inhibitors, particularly in patients with DM. The risk of HF was not different between these two groups.

Disclosure of Interest: None declared

DOI: 10.1136/annrheumdis-2017-eular.2407

\section{SAT0090 SUCCESS RATE OF BLOOD PRESSURE GOAL ACHIEVEMENT IN INFLAMMATORY JOINT DISEASES}

S. Rollefstad, P. Norheim, E. Ikdahl, G. Wibetoe, A.G. Semb. Preventive Cardio-Rheuma Clinic, Department of Rheumatology, Diakonhjemmet Hospital, Oslo, Norway

Background: The excess risk of cardiovascular disease (CVD) in patients with inflammatory joint diseases (IJD) is attributable to several risk factors, including a high prevalence of hypertension. However, there is limited knowledge on the effect of antihypertensive treatment (a-HTT) in these patients.

Objectives: Our objective was to initiate a-HTT when indicated and treat to guideline recommended blood pressure (BP) goal in IJD patients. We also aimed to evaluate the effect of a-HTT in this patient population, and which factors were associated with BP goal attainment.

Methods: Patients with IJD ( $n=765)$ were referred from a rheumatology outpatient clinic or general practitioners to a preventive cardio-rheuma clinic. All patients underwent a CVD risk evaluation, including BP measurements (performed using and Omron M7 apparatus). Antihypertensive treatment was initiated in accordance with guidelines, and the BP treatment goal was $<140 / 90 \mathrm{mmHg}$.

Results: Of the 765 IJD patients referred (rheumatoid arthritis $n=450$, ankylosing spondylitis $n=210$ and psoriatic arthritis $n=105), 104(13.6 \%)$ had an indication for BP lowering, while 224 (29.3\%) were already using a-HTT at the first consultation. For those where a-HTT was initiated at baseline $(n=104)$, there was a highly significant change in BP from first to final consultation (Fig 1a). BP goal was achieved in $84(80.8 \%$ ) patients (Fig 1b), using mean \pm SD $3.1 \pm 1.7$ consultations. Dose adjustments was done in $38(36.5 \%)$ of the patients with median (IQR) a-HTT dose adjustments of $1(1,1.25)$. In $9(8.7 \%)$ patients the 